\title{
Baryon octet distribution amplitudes in Wandzura-Wilczek approximation
}

\author{
I. V. Anikin ${ }^{1,2}$ and A. N. Manashov ${ }^{3,2}$ \\ ${ }^{1}$ Bogoliubov Laboratory of Theoretical Physics, JINR, 141980 Dubna, Russia \\ ${ }^{2}$ Institut für Theoretische Physik, Universität Regensburg, D-93040 Regensburg, Germany \\ ${ }^{3}$ Institut für Theoretische Physik, Universität Hamburg, D-22761 Hamburg, Germany
}

(Received 23 December 2015; published 10 February 2016)

\begin{abstract}
We study higher-twist distribution amplitudes for the $S U_{F}(3)$ baryon octet. We identify independent functions for all baryons in the isospin symmetry limit and calculate the Wandzura-Wilczek contributions to the twist-4 and -5 distribution amplitudes.
\end{abstract}

DOI: 10.1103/PhysRevD.93.034024

\section{INTRODUCTION}

Hard exclusive processes give us a unique possibility to study the internal structure of hadrons. The theoretical description of exclusive processes is based on the QCD factorization approach [1-6]. Scattering amplitudes (decay amplitudes) in this approach are given by convolution of the coefficient function which can be calculated perturbatively with nonperturbative functions - the distribution amplitudes (DAs). In the infinite momentum frame DAs can be interpreted as the momentum fraction distributions of partons in hadrons.

The DAs are usually classified according to their twist. In the QCD factorization approach, where the relevant $Q^{2}$ is large, the dominant contributions to an amplitude come from DAs of lowest possible twist. The highertwist DAs give rise to the power-suppressed corrections. The factorization approach works quite well for the mesons but for baryons it encounters conceptual difficulties. Namely, it has been shown in Refs. [7-10] that the soft rescattering processes produce contributions that break the factorization theorems; see for a detailed discussion Refs. [11-13].

A quantitative description of the nucleon electromagnetic form factors has been achieved in the framework of the light-cone rules [14-16] by taking into account the power-suppressed [17-20] and next-to-leading-order $[21,22]$ corrections. As it has been shown, the powersuppressed corrections, which are parameterized by the higher-twist nucleon DAs, give a sizable contribution for moderate $Q^{2} \sim 2-5 \mathrm{GeV}$.

Unfortunately, our knowledge of the nucleon (baryon) DAs is quite limited. Only the leading-twist nucleon DA is known with some degree of certainty, while the estimates of the higher-twist nucleon DAs are very poor (see e.g. Refs. [18,19,22,23], and references therein).

At the same time, the higher-twist DAs contain the contributions that are related to the lower-twist DAs- the so-called Wandzura-Wilczek (WW) contributions [24]. For mesons, the genuine higher-twist DAs often appear to be smaller than the corresponding WW terms (see, for example, $[25,26])$. In many cases, keeping only the WW terms one gets a quite good approximation for the highertwist DAs (the so-called WW approximation).

The lowest-twist meson DAs are defined by matrix elements of two-particle (quark-antiquark or gluon) light-ray operators. For such operators the WW contributions were calculated long ago. A detailed discussion of the method can be found in Ref. [25]. We also mention that WW corrections to the generalized parton distributions have been derived in [27-31] and the relevant problem of WW contributions has been considered in Refs. [32-37].

The situation with baryon DAs is more complicated because they are determined by matrix elements of threeparticle (three-quark) operators. Until now the WW corrections were only known for the first few moments of the nucleon DAs [17,18].

The effective technique that allows one to calculate the WW terms for the multiparticle DAs was developed in $[38,39]$. The approach is based on the spinor formalism and conformal wave expansion for the light-ray operators. Using this technique the WW corrections to the three-particle nucleon DAs were calculated up to twist 5 [38,39].

In the recent paper [40] the first results of the lattice calculation of the baryon octet DAs moments have been presented. Keeping in mind ongoing progress in lattice calculations, one can expect that information on highertwist DAs will be available soon. Therefore, in the present paper, we derive the WW corrections to the higher-twist baryon octet DAs.

The paper is organized as follows: in Sec. II we recall the basics of the spinor formalism and fix our notations. In Sec. III, we give the definitions of the DAs for the baryon octet. Sections III A and III B contain the analysis of the mixed chirality DAs up to twist 5 and in Sec. III C we consider the chiral DAs of twist 4 and 5. The Appendixes contain the $S U_{F}(3)$ relations between different DAs and explicit expressions for the few first polynomials entering the expansion of baryon DAs. 


\section{SPINOR CONVENTIONS}

We closely follow the notations of Ref. [41]. In the Weyl representation, the Dirac $\gamma$ matrices take the following form:

$$
\gamma^{\mu}=\left(\begin{array}{cc}
0 & \sigma^{\mu} \\
\bar{\sigma}^{\mu} & 0
\end{array}\right)
$$

where

$$
\sigma^{\mu}=(I, \overrightarrow{\boldsymbol{\sigma}}), \quad \bar{\sigma}^{\mu}=(I,-\overrightarrow{\boldsymbol{\sigma}}),
$$

and $\vec{\sigma}$ are the Pauli matrices. The $\gamma_{5}$ matrix and the charge conjugation matrix $C=i \gamma^{2} \gamma^{0}$ are defined, respectively, as

$$
\gamma_{5}=\left(\begin{array}{cc}
-I & 0 \\
0 & I
\end{array}\right), \quad C=\left(\begin{array}{cc}
i \sigma^{2} & 0 \\
0 & -i \sigma^{2}
\end{array}\right) .
$$

The Dirac spinor is constructed from two Weyl spinors:

$$
q=\left(\begin{array}{c}
q^{\downarrow} \\
q^{\uparrow}
\end{array}\right)=\left(\begin{array}{c}
\psi_{\alpha} \\
\bar{\chi}^{\dot{\alpha}}
\end{array}\right) .
$$

Here $q^{\downarrow(\uparrow)}=\frac{1}{2}\left(1 \mp \gamma_{5}\right) q$ are the left- (right-) handed quark fields, respectively.

An arbitrary vector $a_{\mu}$ can be mapped to a $2 \times 2$ matrix as

$$
a_{\alpha \dot{\alpha}}=a_{\mu} \sigma_{\alpha \dot{\alpha}}^{\mu}=\left(\begin{array}{cc}
a_{0}-a_{3} & -a_{1}+i a_{2} \\
-a_{1}-i a_{2} & a_{0}+a_{3}
\end{array}\right) .
$$

In the studies of hard processes, the lightlike vectors $n$ and $\bar{n}\left[n^{2}=\bar{n}^{2}=0,(n \cdot \bar{n}) \neq 0\right]$ are usually introduced. They can be parameterized by two auxiliary Weyl spinors $\lambda$ and $\mu$ as

$$
n_{\alpha \dot{\alpha}}=\lambda_{\alpha} \bar{\lambda}_{\dot{\alpha}}, \quad \tilde{n}_{\alpha \dot{\alpha}}=\mu_{\alpha} \bar{\mu}_{\dot{\alpha}}
$$

The rules for rising (lowering) spinor indices read

$$
\begin{array}{ll}
\lambda^{\alpha}=\epsilon^{\alpha \beta} \lambda_{\beta}, & \lambda_{\alpha}=\lambda^{\beta} \epsilon_{\beta \alpha}, \\
\bar{\lambda}^{\dot{\alpha}}=\bar{\lambda}_{\dot{\beta}} \bar{\epsilon}^{\dot{\beta} \dot{\alpha}}, & \bar{\lambda}_{\dot{\alpha}}=\bar{\epsilon}_{\dot{\alpha} \dot{\beta}} \bar{\lambda}^{\dot{\beta}},
\end{array}
$$

where the antisymmetric Levi-Civita tensors are normalized as $\epsilon_{12}=\epsilon^{12}=-\bar{\epsilon}_{i \dot{2}}=-\bar{\epsilon}^{i \dot{2}}=1$. The products of Weyl spinors are defined as

$$
(\lambda \mu)=\lambda^{\alpha} \mu_{\alpha}=-(\mu \lambda), \quad(\bar{\lambda} \bar{\mu})=\bar{\lambda}_{\dot{\alpha}} \bar{\mu}^{\dot{\alpha}}=-(\bar{\mu} \bar{\lambda}) .
$$

In the following we use shorthand notation for the quark field projections onto the auxiliary spinors:

$$
\begin{aligned}
& q_{+}^{\downarrow}=\left(\lambda q^{\downarrow}\right), \quad q^{\downarrow}=\left(\mu q^{\downarrow}\right), \\
& q_{+}^{\uparrow}=\left(\bar{\lambda} q^{\uparrow}\right), \quad q_{-}^{\uparrow}=\left(\bar{\mu} q^{\uparrow}\right) \text {. }
\end{aligned}
$$

The quark fields can be written in the form

$$
(\mu \lambda) q_{\alpha}^{\downarrow}=\lambda_{\alpha} q_{-}^{\downarrow}-\mu_{\alpha} q_{+}^{\downarrow}, \quad(\bar{\lambda} \bar{\mu}) q_{\dot{\alpha}}^{\uparrow}=\bar{\lambda}_{\dot{\alpha}} q_{-}^{\uparrow}-\bar{\mu}_{\dot{\alpha}} q_{+}^{\uparrow} .
$$

Constructing the light-ray operators it is useful to ascribe two quantum numbers-twist and helicity- to the quark field projections (8). The plus components, $q_{+}^{\downarrow(\uparrow)}$, have the collinear twist equal to 1 , while the minus components, $q_{-}^{\downarrow}(\uparrow)$, have twist 2 . The helicity of the $q_{+}^{\downarrow}, q_{-}^{\uparrow}$ projections is $-\frac{1}{2}$, whereas $q_{+}^{\uparrow}, q_{-}^{\downarrow}$ have helicity $+\frac{1}{2}$; see Ref. [38] for details. We recall here that the collinear twist is defined as the difference of the canonical dimension and spin projection on the light-cone direction.

\section{BARYON OCTET DISTRIBUTION AMPLITUDES}

\section{A. Twist-3 distribution amplitudes}

In this paper we study the three-quark distribution amplitudes of the baryon octet,

$$
B=\left(\begin{array}{ccc}
\frac{\Lambda^{0}}{\sqrt{6}}+\frac{\Sigma^{0}}{\sqrt{2}} & \Sigma^{+} & p \\
\Sigma^{-} & \frac{\Lambda^{0}}{\sqrt{6}}-\frac{\Sigma^{0}}{\sqrt{2}} & n \\
\Xi^{-} & \Xi^{0} & -\frac{2}{\sqrt{6}} \Lambda^{0}
\end{array}\right),
$$

in the isospin symmetry limit. It is helpful to recall the quark content of the isospin multiplets: $(p, n) \sim$ $($ uud,$u d d), \quad\left(\Sigma^{+}, \Sigma^{0}, \Sigma^{-}\right) \sim(u u s, u d s, d d s), \quad\left(\Xi^{0}, \Xi^{-}\right) \sim$ (uss, dss) and $\Lambda^{0} \sim u d s$.

The leading-twist distribution amplitudes are defined by the matrix elements of the twist- 3 three-quark operator:

$$
\mathbb{O}_{3, \vec{m}}^{\downarrow}(\mathbf{z})=\epsilon^{i j k} q_{+, m_{1}}^{\downarrow, i}\left(z_{1}\right) q_{+, m_{2}}^{\uparrow, j}\left(z_{2}\right) q_{+, m_{3}}^{\downarrow, k}\left(z_{3}\right) .
$$

Here $\mathbf{z}=\left\{z_{1}, z_{2}, z_{3}\right\}, \vec{m}=\left\{m_{1}, m_{2}, m_{3}\right\} . m_{k}$ are the flavor indices, $m_{k}=u, d, s$ and $i, j, k$ are the color indices. The matrix element of (11) takes the form

$$
\left\langle 0\left|\mathbb{O}_{3, \vec{m}}^{\downarrow}(\mathbf{z})\right| B\right\rangle=-\frac{p_{+}}{2}\left(u_{B}\right)_{+}^{\downarrow} \int \mathcal{D} x e^{-i p_{+} \sum x_{i} z_{i}} \Phi_{3, \vec{m}}^{(B)}(x),
$$

where $\Phi_{3, \vec{m}}^{(B)}(x)$ is the corresponding DA, $u_{B}$ stands for the Dirac spinor of the baryon $B, p$ denotes its momentum, $p_{+}=(p n)$ and the integration measure is defined as follows:

$$
\mathcal{D} x=d x_{1} d x_{2} d x_{3} \delta\left(1-x_{1}-x_{2}-x_{3}\right) .
$$


It is clear that the matrix element is nonzero only if the flavor indices of the operator match the flavor content of the baryon. Invariance of the operator under the permutation $\left(z_{1}, m_{1}\right) \leftrightarrow\left(z_{3}, m_{3}\right)$ together with the isospin symmetry allows one to express all DAs in terms of seven independent functions. We choose them as

$$
\begin{array}{cccc}
\Phi_{3, \text { uиd }}^{(p)} & \Phi_{3, \text { uиs }}^{\left(\Sigma^{+}\right)} & \Phi_{3, \text { ssu }}^{\left(\Xi^{0}\right)} & \Phi_{3, \text { uds }}^{(\Lambda)} \\
& \Phi_{3, \text { usu }}^{\left(\Sigma^{+}\right)} & \Phi_{3, \text { sus }}^{\left(\Xi^{0}\right)} & \Phi_{3, \text { usd }}^{(\Lambda)}
\end{array}
$$

We mark the DAs by their positions in this table and do not specify the flavor indices explicitly; i.e. $\Phi_{3}^{(B, k)}(x)$ denote the DAs of the baryon $B$ in the $k$ th row $(k=1,2)$.

One can easily see that the functions in the second row have certain parity with respect to $x_{1} \leftrightarrow x_{3}$ permutations. Namely, the DAs $\Phi_{3}^{\left(\Sigma^{+}, 2\right)}(x)$ and $\Phi_{3}^{\left(\Xi^{0}, 2\right)}(x)$ are symmetric while $\Phi_{3}^{(\Lambda, 2)}(x)$ is antisymmetric under this permutation. The DAs in the first row, $\Phi_{3}^{(B, 1)}(x)$, do not possess any symmetries under permutations of the arguments. The proton DAs, $\Phi_{3}^{(p, 2)}=\Phi_{3, u d u}^{(p)}$, in the second row are absent because this function is not independent and can be expressed in term of $\Phi_{3}^{(p, 1)}$, namely,

$\Phi_{3}^{(p, 2)}(x)=-\Phi_{3}^{(p, 1)}\left(x_{1}, x_{2}, x_{3}\right)-\Phi_{3}^{(p, 1)}\left(x_{3}, x_{2}, x_{1}\right)$.

The DAs (14) are related to the vector, axial-vector and tensor twist-3 DAs, $V_{1}, A_{1}, T_{1}$ which appear in the decomposition of the three-quark operator with open spinor indices [23]:

$$
\begin{aligned}
& { }^{4}\left\langle 0\left|\epsilon^{i j k} q_{m_{1} \alpha}^{i}\left(z_{1} n\right) q_{m_{2} \beta}^{j}\left(z_{2} n\right) q_{m_{3} \gamma}^{k}\left(z_{3} n\right)\right| B(p)\right\rangle \\
& \quad=v_{\alpha \beta, \gamma} V_{1}^{B}(\vec{z})+a_{\alpha \beta, \gamma} A_{1}^{B}(\vec{z})+t_{\alpha \beta, \gamma} T_{1}^{B}(\vec{z})+\ldots,
\end{aligned}
$$

where the dots stand for higher-twist terms and

$$
\begin{aligned}
v_{\alpha \beta, \gamma} & =(p C)_{\alpha \beta}\left(\gamma_{5} u^{+}(P)\right)_{\gamma}, \\
a_{\alpha \beta, \gamma} & =\left(p \gamma_{5} C\right)_{\alpha \beta}\left(u^{+}(P)\right)_{\gamma}, \\
t_{\alpha \beta, \gamma} & =\left(i \sigma_{\mu p}^{\perp} C\right)_{\alpha \beta}\left(\gamma_{\perp}^{\mu} \gamma_{5} u^{+}(P)\right)_{\gamma} .
\end{aligned}
$$

As usual, the DAs in momentum space are defined by the Fourier transform

$$
F(\vec{z})=\int \mathcal{D} x e^{-i(p n) \sum x_{i} z_{i}} F(x) .
$$

Projecting both sides of Eq. (16) onto the auxiliary spinors $\lambda, \bar{\lambda}$ one derives

$$
\Phi_{3}^{(B, 1)}(x)=V_{1}^{B}(x)-A_{1}^{B}(x)
$$

and [cf. Eq. (15)]

$$
\Phi_{3}^{(B, 2)}\left(x_{1}, x_{2}, x_{3}\right)=-2 T_{1}^{B}\left(x_{1}, x_{3}, x_{2}\right) .
$$

The conformal wave expansion of the DAs (14) goes over polynomials $P_{N q}$ which are solutions of the renormalization group $(\mathrm{RG})$ equations,

$$
\Phi_{3}^{(B, k)}(x, \mu)=x_{1} x_{2} x_{3} \sum_{N, q} \phi_{N q}^{(B, k)}(\mu) P_{N q}(x),
$$

where $\mu$ is the renormalization scale. The common prefactor is fixed by the conformal spins of the fields. The functions $P_{N q}(x)$ are the homogeneous polynomials of degree $N, P_{N q}(s x)=s^{N} P_{N q}(x)$, that form an orthogonal system

$$
\int \mathcal{D} x x_{1} x_{2} x_{3} P_{N q}(x) P_{N q^{\prime}}^{\dagger}(x)=\delta_{q q^{\prime}} c_{N q}^{-1} .
$$

The index $q$ enumerates different polynomials of the same degree. The polynomials $P_{N q}$ can be obtained as solutions of the one-loop RG equation for twist-3 three quark operators. Note that these polynomials do not depend on the flavor content of the three-quark operator because the evolution kernels are flavor-blind. Each polynomial $P_{N q}$ is either symmetric or antisymmetric under permutations of the first and third arguments:

$$
P_{N q}^{( \pm)}\left(x_{1}, x_{2}, x_{3}\right)= \pm P_{N q}^{( \pm)}\left(x_{3}, x_{2}, x_{1}\right) .
$$

The reduced matrix element $\phi_{N q}^{(B, k)}$ can be expressed as a convolution integral:

$$
\phi_{N q}^{(B, k)}=c_{N q} \int \mathcal{D} x P_{N q}^{\dagger}(x) \Phi_{3}^{(B, k)}(x) .
$$

To the one-loop accuracy, the reduced matrix elements $\phi_{N q}(\mu)$ have an autonomous scale dependence. Evidently, for the DAs $\Phi_{3}^{\left(\Xi^{0}, 2\right)}, \Phi_{3}^{\left(\Sigma^{+}, 2\right)}$ and $\Phi_{3}^{(\Lambda, 2)}$ the sum in (27) goes over the symmetric (antisymmetric) polynomials only. The first few polynomials with the corresponding anomalous dimensions are given in Appendix B. More details can be found in Refs. [38,42-44].

For later convenience we split the DAs $\Phi_{3}^{(B, 1)}(x)$ into symmetric and antisymmetric parts with respect to $x_{1} \leftrightarrow x_{3}$ permutation:

$$
\Phi_{3}^{(B, 1)}(x)=\Phi_{3}^{(B, 1+)}(x)+\Phi_{3}^{(B, 1-)}(x)
$$

and introduce the notation $\Phi_{3}^{(B, A)}(x)$, where the label $A$ takes the values $A=\{1 \pm, 2\}$. Let us assign the signature factor $\varkappa(B, A)$ :

$$
\varkappa(B, 1 \pm)= \pm, \quad \varkappa(B \neq \Lambda, 2)=+, \quad \varkappa(\Lambda, 2)=-
$$


to each DA. The signature factor defines parity of the polynomials $P_{N q}^{ \pm}$that enter the conformal expansion for the corresponding DA, namely,

$$
\Phi_{3}^{(B, A)}(x, \mu)=x_{1} x_{2} x_{3} \sum_{N, q} \phi_{N q}^{(B, A)}(\mu) P_{N q}^{\chi(B, A)}(x) .
$$

As we see in the next section the higher-twist DAs can be written in a quite similar form.

\section{B. Mixed chirality higher-twist distribution amplitudes}

We define the higher-twist three-quark operators of mixed chirality as

$$
\begin{aligned}
& \mathbb{O}_{4, \vec{m}}^{\uparrow}(\mathbf{z})=q_{+, m_{1}}^{\downarrow}\left(z_{1}\right) q_{+, m_{2}}^{\uparrow}\left(z_{2}\right) q_{-, m_{3}}^{\downarrow}\left(z_{3}\right), \\
& \mathbb{O}_{5, \vec{m}}^{\downarrow}(\mathbf{z})=q_{-, m_{1}}^{\downarrow}\left(z_{1}\right) q_{-, m_{2}}^{\uparrow}\left(z_{2}\right) q_{+, m_{3}}^{\downarrow}\left(z_{3}\right),
\end{aligned}
$$

where it is tacitly assumed that color indices are contracted with the antisymmetric tensor. We also recall that $q_{-}^{\downarrow} \equiv\left(\mu q^{\downarrow}\right), q_{-}^{\uparrow} \equiv\left(\bar{\mu} q^{\uparrow}\right)$. The operators $\mathbb{O}_{4, \vec{m}}^{\uparrow}$ and $\mathbb{O}_{5, \vec{m}}^{\downarrow}$ have helicities $+1 / 2$ and $-1 / 2$, respectively.

In the proton case matrix elements of these operators define the functions $\Phi_{4}, \Psi_{4}$ and $\Phi_{5}, \Psi_{5}$ [23]. For all other baryons in the octet (except neutron) there are three independent DAs related to the matrix elements of these operators.

Twist-4 and -5 DAs can be defined as

$$
\begin{aligned}
\left\langle 0\left|\mathbb{O}_{4, \vec{m}}^{\uparrow}(\mathbf{z})\right| B\right\rangle & =\frac{m_{B}}{4}(\mu \lambda)\left(u_{B}^{\uparrow}\right)_{+} \int \mathcal{D} x e^{-i p_{+} \sum z_{k} x_{k}} \Phi_{4, \vec{m}}^{(B)}(x), \\
\left\langle 0\left|\mathbb{O}_{5, \vec{m}}^{\downarrow}(\mathbf{z})\right| B\right\rangle & =-\frac{m_{B}}{4}(\mu \lambda)\left(u_{B}^{\uparrow}\right)_{-} \int \mathcal{D} x e^{-i p_{+} \sum z_{k} x_{k}} \Phi_{5, \vec{m}}^{(B)}(x) .
\end{aligned}
$$

There are altogether 11 independent DAs for the $p, \Sigma^{+}, \Xi^{0}$ and $\Lambda$ baryons for each twist $t=4,5$ :

$$
\begin{array}{cccc}
\Phi_{t, \text { uud }}^{(p)} & \Phi_{t, \text { uus }}^{\left(\Sigma^{+}\right)} & \Phi_{t, \text { ssu }}^{\left(\Xi^{0}\right)} & \Phi_{t, \text { uds }}^{(\Lambda)} \\
& \Phi_{t, \text { usu }}^{\left(\Sigma^{+}\right)} & \Phi_{t, \text { sus }}^{\left(\Xi^{0}\right)} & \Phi_{t, \text { usd }}^{(\Lambda)} \\
\Phi_{t, \text { duu }}^{(p)} & \Phi_{t, \text { suu }}^{\left(\Sigma^{+}\right)} & \Phi_{t, \text { uss }}^{\left(\Xi^{0}\right)} & \Phi_{t, \text { sdu }}^{(\Lambda)}
\end{array}
$$

Similar to the twist-3 case we introduce the notation $\Phi_{t}^{(B, A)}(x)$, with the index $A$ taking the values $(1 \pm, 2)$. The function $\Phi_{t}^{(B, 2)}(x)$ stands for the DA in the second row of Table (30) while $\Phi_{t}^{(B, 1 \pm)}$ is given by the combination of DAs in the first and last row, namely,

$$
\Phi_{t}^{(B, 1 \pm)}(x)=\frac{1}{2}\left(\Phi_{t}^{(B, 1)}(x) \pm \Phi_{t}^{(B, 3)}(x)\right) .
$$

We want to stress here that contrary to the twist-3 functions, the higher-twist functions $\Phi_{t}^{(B, A)}(x)$ are not symmetric under the argument permutation.

In this notation the conventional proton DAs of twist 4 and 5 [23] take the following form:

$$
\begin{aligned}
& \Phi_{4(5)}(x)=\Phi_{4(5)}^{(p, 1+)}\left(x_{1}, x_{2}, x_{3}\right)+\Phi_{4(5)}^{(p, 1-)}\left(x_{1}, x_{2}, x_{3}\right), \\
& \Psi_{4(5)}(x)=\Phi_{4(5)}^{(p, 1+)}\left(x_{3}, x_{1}, x_{2}\right)-\Phi_{4(5)}^{(p, 1-)}\left(x_{3}, x_{1}, x_{2}\right) .
\end{aligned}
$$

The higher-twist DAs $\Phi_{t}^{(B, A)}(x)$ contain contributions from both the operators of the geometrical twist- $t$ (we recall that the geometrical twist is defined as $t=\ell^{\text {can }}-s$, where $\ell^{\text {can }}$ is the canonical scaling dimension of an operator and $s$ is its Lorentz spin) and the lower-twist operators. Therefore, we represent the higher-twist DAs in the following form:

$$
\begin{aligned}
& \Phi_{4}^{(B, A)}(x)=\Phi_{4}^{(B, A), g}(x)+\Phi_{4}^{(B, A), W W_{3}}(x), \\
& \Phi_{5}^{(B, A)}(x)=\Phi_{5}^{(B, A), g}(x)+\Phi_{5}^{(B, A), W W_{3}}(x)+\Phi_{5}^{(B, A), W W_{4}}(x) .
\end{aligned}
$$

The conformal wave expansion of the genuine twist-4 DAs, $\Phi_{4}^{(B, A), g}(x)$, takes the form

$$
\Phi_{4}^{(B, A), g}(x)=x_{1} x_{2} \sum_{N q} \eta_{N q}^{(B, A)}(\mu) R_{N q}^{\varkappa(B, A)}(x),
$$

where the signature factor $\varkappa(B, A)$ is defined in (26).

The polynomials $R_{N q}^{( \pm)}$are the eigenfunctions of the evolution kernel for twist-4 operators; see Ref. [38] for a general discussion. Index $N$ stands for a degree of the polynomial $R_{N q}^{( \pm)}(s x)=s^{N} R_{N q}^{( \pm)}(s x)$ and $q$ enumerates different polynomials of the same degree. Several lowest polynomials with the corresponding anomalous dimensions can be found in Appendix B. Here, we note that there exists only one polynomial of the degree $N=0, R_{00}^{(-)}=1$. Therefore, the expansion of the positive signature DAs, i.e. $\Phi_{4}^{(B, A)}$ with $\varkappa(B, A)=+$, goes over the polynomials of degree $N \geq 1$.

The genuine twist-5 distributions have a similar expansion:

$$
\Phi_{5}^{(B, A) g}(x)=x_{3} \sum_{N \geq 1, q} \zeta_{N q}^{(B, A)}(\mu) T_{N q}^{\varkappa(B, A)}(x) .
$$

The form of this expansion is dictated by the flavor blindness of the RG equations for operators (28). We emphasize, however, that neither the polynomials $T_{N q}^{ \pm}$nor the anomalous dimensions have been calculated so far.

The structure of the WW contributions to the DAs (33) and (34) does not depend on the flavor content of baryons and can be easily extracted from the results of Ref. [39]. We derive 


$$
\begin{aligned}
\Phi_{4}^{(B, A) W W}(x)= & -x_{1} x_{2} \sum_{N q} \frac{\phi_{N q}^{(B, A)}(\mu)}{(N+2)(N+3)} \\
& \times\left[N+2-\partial_{x_{3}}\right] x_{3} P_{N q}^{\chi(B, A)}(x),
\end{aligned}
$$

where summation goes over the polynomials of the parity $\varkappa(B, A)$.

For the twist- 5 DAs, the WW terms of twist 3 and twist 4 have the form

$$
\begin{aligned}
\Phi_{5}^{(B, A) W W_{3}}(x)= & x_{3} \sum_{N q} \frac{\phi_{N q}^{(B, A)}(\mu)}{(N+2)(N+3)}\left[-(N+2)^{2}\right. \\
& \left.+\left(N+1-\partial_{x_{2}}\right)\left(N+2-\partial_{x_{1}}\right)\right] \\
& \times x_{1} x_{2} P_{N q}^{\varkappa(B, A)}(x)
\end{aligned}
$$

and

$$
\begin{aligned}
\Phi_{5}^{(B, A) W W_{4}}(x)= & x_{3} \sum_{N q} \frac{\eta_{N q}^{(B, A)}(\mu)}{(N+1)(N+3)} \\
& \times\left[N+1-\partial_{x_{2}}\right] x_{2} R_{N q}^{\chi(B, A)}\left(x_{3}, x_{2}, x_{1}\right),
\end{aligned}
$$

respectively.

\section{Chiral distribution amplitudes}

We now define the chiral three-quark operators as

$$
\begin{aligned}
& \mathbb{O}_{4, \vec{m}}^{\downarrow}(\mathbf{z})=q_{-, m_{1}}^{\downarrow}\left(z_{1}\right) q_{+, m_{2}}^{\downarrow}\left(z_{2}\right) q_{+, m_{3}}^{\downarrow}\left(z_{3}\right), \\
& \mathbb{O}_{5, \vec{m}}^{\uparrow}(\mathbf{z})=q_{+, m_{1}}^{\downarrow}\left(z_{1}\right) q_{-, m_{2}}^{\downarrow}\left(z_{2}\right) q_{-, m_{3}}^{\downarrow}\left(z_{3}\right) .
\end{aligned}
$$

They are constructed from the left-handed chiral quarks and they have the helicity $-1 / 2$ and $+1 / 2$, respectively. The DAs related to these operators are defined by

$$
\begin{aligned}
\left\langle 0\left|\mathbb{O}_{4, \vec{m}}^{\downarrow}(\mathbf{z})\right| B\right\rangle & =\frac{m_{B}}{4}(\mu \lambda)\left(u_{B}^{\downarrow}\right)_{+} \int \mathcal{D} x e^{-i p_{+} \sum z_{k} x_{k}} \Xi_{4, \vec{m}}^{(B)}(x), \\
\left\langle 0\left|\mathbb{O}_{5, \vec{m}}^{\downarrow}(\mathbf{z})\right| B\right\rangle & =-\frac{m_{B}}{4}(\mu \lambda)\left(u_{B}^{\downarrow}\right)_{-} \int \mathcal{D} x e^{-i p_{+} \sum z_{k} x_{k}} \Xi_{5, \vec{m}}^{(B)}(x) .
\end{aligned}
$$

For each twist, there are 7 independent DAs which can be chosen as follows:

$$
\begin{array}{cccc}
\Xi_{t, \text { uud }}^{(p)} & \Xi_{t, \text { uus }}^{\left(\Sigma^{+}\right)} & \Xi_{t, \text { ssu }}^{\left(\Xi^{0}\right)} & \Xi_{t, \text { uds }}^{(\Lambda)} \\
& \Xi_{t, \text { suu }}^{\left(\Sigma^{+}\right)} & \Xi_{t, \text { uss }}^{\left(\Xi^{0}\right)} & \Xi_{t, \text { sdu }}^{(\Lambda)}
\end{array}
$$

Similarly to the previous case, we denote the DA of the baryon $B$ in the $k$ th row (see the table) by $\Xi_{t}^{(B, k)}$. The proton DAs coincide with the conventional definitions of
Ref. [23], i.e. $\Xi_{t}^{(p, 1)}(x)=\Xi_{t}(x)$. The first two DAs in the second row, $\Xi_{t}^{\left(\Sigma^{+}, 2\right)}(x)$ and $\Xi_{t}^{\left(\Xi^{0}, 2\right)}(x)$, are obviously symmetric under interchange of the two last arguments, while the $\Xi_{t}^{(\Lambda, 2)}(x)$ is antisymmetric under $x_{2} \leftrightarrow x_{3}$. The nucleon DA $\Xi_{t}^{(p, 2)}$ is not independent:

$$
\Xi_{t}^{(p, 2)}(x)=-\left(1+P_{23}\right) \Xi_{t}^{(p, 1)}(x),
$$

and, therefore, it is not listed in the table.

The conformal wave expansion for the nucleon DA $\Xi_{4}^{(p, 1)}$ was worked out in Ref. [38]. The eigenfunctions of the evolution kernel for the higher-twist chiral threequark operators are characterized by parity (which is $\left.\varepsilon=\left\{1, e^{ \pm i 2 \pi / 3}\right\}\right)$ under the combined cyclic permutations in the position and flavor spaces. The conformal expansion of the nucleon chiral DA, $\Xi_{4}^{(p, 1)}$, goes over the certain combination of the eigenfunctions with parity $e^{ \pm i 2 \pi / 3}$. The expansion of the chiral DAs for the other baryons involves the eigenfunctions of the parity $\varepsilon=1$ as well. A more detailed discussion can be found in Ref. [38]. Here, we present the final results only. The expansions for the $\Lambda$ baryon as compared to all other baryons in the octet have a slightly different form. Namely,

$$
\begin{aligned}
& \Xi_{4}^{(B \neq \Lambda, k)}(x) \\
& \quad=x_{2} x_{3}\left[\sum_{N q} \xi_{N q}^{(B)}(\mu) \Pi_{N q}^{(k)}(x)+\sum_{N q} \theta_{N q}^{(B)}(\mu) \Pi_{N q}^{(+)}(x)\right], \\
& \Xi_{4}^{(\Lambda, k)}(x) \\
& \quad=x_{2} x_{3}\left[\sum_{N q} \xi_{N q}^{(\Lambda)}(\mu) \Lambda_{N q}^{(k)}(x) \pm \sum_{N q} \theta_{N q}^{(\Lambda)}(\mu) \Lambda_{N q}^{(-)}(x)\right] .
\end{aligned}
$$

In the last formula, the signs " \pm " correspond to $k=1,2$, respectively. The polynomials $\Pi_{N q}^{(k)}(x), \Lambda_{N q}^{(k)}(x)$ are related to the eigenfunctions $\Pi_{N q}(x)$ of the parity $\epsilon=e^{ \pm i 2 \pi / 3}$, which were calculated in Ref. [38],

$$
\begin{aligned}
& \Pi_{N q}^{(1)}(x)=\Pi_{N q}(x), \\
& \Pi_{N q}^{(2)}(x)=-\left(1+P_{23}\right) \Pi_{N q}(x), \\
& \Lambda_{N q}^{(1)}(x)=\left(1+2 P_{23}\right) \Pi_{N q}(x), \\
& \Lambda_{N q}^{(2)}(x)=-\left(1-P_{23}\right) \Pi_{N q}(x),
\end{aligned}
$$

where $P_{23}$ is the permutation operator:

$$
P_{23} f\left(x_{1}, x_{2}, x_{3}\right)=f\left(x_{1}, x_{3}, x_{2}\right) \text {. }
$$

The eigenfunctions $\tilde{\Pi}_{N q}^{(+)}$and $\Lambda_{N q}^{(-)}$belong to the parity sector $\epsilon=1$ and they are (anti)symmetric under $x_{2} \leftrightarrow x_{3}$ permutation: 


$$
\begin{gathered}
P_{23} \Pi_{N q}^{(+)}(x)=\Pi_{N q}^{(+)}(x), \\
P_{23} \Lambda_{N q}^{(-)}(x)=-\Lambda_{N q}^{(-)}(x) .
\end{gathered}
$$

Notice that for the proton $\theta_{N q}^{(p)}(\mu)=0$ by symmetry.

In order to write down the WW contributions to the twist-5 amplitudes, we define the following functions:

$$
\begin{aligned}
W^{(B)}(x)= & -P_{12} \sum_{N q} \frac{\xi_{N q}^{(B)}(\mu)}{(N+1)(N+3)}\left[\left(N+1-\partial_{3}\right) x_{3} \Pi_{N q}^{(1)}(x)\right. \\
& \left.+\left(N+1-\partial_{1}\right) x_{1} \Pi_{N q}^{(2)}(x)\right], \\
W^{(\Lambda)}(x)= & P_{12} \sum_{N q} \frac{\xi_{N q}^{(\Lambda)}(\mu)}{(N+1)(N+3)}\left[\left(N+1-\partial_{3}\right) x_{3} \Lambda_{N q}^{(1)}(x)\right. \\
& \left.+\left(N+1-\partial_{1}\right) x_{1} \Lambda_{N q}^{(2)}(x)\right],
\end{aligned}
$$

and

$$
\begin{aligned}
W_{+}^{(B)}(x)= & -P_{12} \sum_{N q} \frac{\theta_{N q}^{(B)}(\mu)}{(N+1)(N+3)}\left[\left(N+1-\partial_{3}\right) x_{3}\right. \\
& \left.+\left(N+1-\partial_{1}\right) x_{1}\right] \Pi_{N q}^{(+)}(x), \\
W_{-}^{(\Lambda)}(x)= & P_{12} \sum_{N q} \frac{\xi_{N q}^{(\Lambda)}(\mu)}{(N+1)(N+3)}\left[\left(N+1-\partial_{3}\right) x_{3}\right. \\
& \left.+\left(N+1-\partial_{1}\right) x_{1}\right] \Lambda_{N q}^{(-)}(x) .
\end{aligned}
$$

With the help of Eqs. (47) and (48), the WW contributions to the twist-5 DA can be written in the following form:

$$
\begin{aligned}
& \Xi_{5}^{(B, 1) W W}(x)=x_{1}\left(W^{(B)}(x)+W_{+}^{(B)}(x)\right), \\
& \Xi_{5}^{(B, 2) W W}(x)=x_{1}\left(-\left(1+P_{23}\right) W^{(B)}(x)+W_{+}^{(B)}(x)\right), \\
& \Xi_{5}^{(\Lambda, 1) W W}(x)=x_{1}\left(W^{(\Lambda)}(x)+W_{-}^{(\Lambda)}(x)\right), \\
& \Xi_{5}^{(\Lambda, 2) W W}(x)=x_{1}\left(\left(1-P_{23}\right) W^{(\Lambda)}(x)-W_{-}^{(\Lambda)}(x)\right) .
\end{aligned}
$$

The first few polynomials $\Pi_{N q}, \Pi_{N q}^{(+)}, \Lambda_{N g}^{(-)}$are given in Appendix B.

\section{ACKNOWLEDGMENTS}

We are grateful to V. M. Braun for useful discussions. The work was supported by the DFG (A. N. M.), Grant No. MO 1801/1-1, and by DAAD (I. V. A.).

\section{APPENDIX A: ISOSPIN RELATIONS}

The isospin symmetry allows one to express DAs for any baryon in the octet in terms of the DAs discussed in the main part of the paper. We recall that the DAs $\Phi_{t, \vec{m}}^{(B)}, \Xi_{t, \vec{m}}^{(B)}$ depend on the flavor indices $m_{1}, m_{2}, m_{3}$ of the quark fields in the corresponding light-ray operators; see Eqs. (12), (29) and (34).

The independent DAs for $p, \Sigma^{+}, \Xi^{0}, \Lambda$ baryons are collected in Tables (14), (30) and (41). Defining distribution amplitudes for the remaining members of the octet we have to specify the flavor indices of the corresponding DAs. For the $\Sigma^{0}$ baryon DAs we simply take over the flavor indices of $\Lambda$ baryon but the corresponding distribution amplitudes differ from each other.

The $n, \Sigma^{-}, \Xi^{-}$baryon DAs inherit flavor indices from the corresponding DAs for $p, \Sigma^{+}, \Xi^{0}$ baryons with the substitution $u \leftrightarrow d$. For example, the twist-3 DAs that have to be added to the first row of Table (14) are $\Phi_{3, d d u}^{(n)}$, $\Phi_{3, d d s}^{\left(\Sigma^{-}\right)}, \Phi_{3, s s d}^{\left(\Xi^{-}\right)}$and $\Phi_{3, u d s}^{\left(\Sigma^{0}\right)}$.

We accept the same phase conventions for the baryons in octet as in Ref. [40] (see Appendix A there). Then the following relations hold:

$$
\begin{aligned}
\operatorname{DA}^{(p)}(x) & =-\mathrm{DA}^{(n)}(x), \\
\mathrm{DA}^{\left(\Xi^{-}\right)}(x) & =-\mathrm{DA}^{\left(\Xi^{0}\right)}(x), \\
\mathrm{DA}^{\left(\Sigma^{+}\right)}(x)=-\mathrm{DA}^{\left(\Sigma^{-}\right)}(x) & =-\sqrt{2} \mathrm{DA}^{\left(\Sigma^{0}\right)}(x),
\end{aligned}
$$

between the DAs of the same type, e.g. [see Table (30)]

$$
\Phi_{5, \text { uus }}^{\left(\Sigma^{+}\right)}(x)=-\Phi_{5, d d s}^{\left(\Sigma^{-}\right)}(x)=-\sqrt{2} \Phi_{5, u d s}^{\left(\Sigma^{0}\right)}(x) .
$$

Below we present the relations between the functions used in the present paper and those introduced in Ref. [40]:

$$
\begin{aligned}
\Phi_{[40] \pm}^{B \neq \Lambda}(x) & =\frac{1}{2}\left(1+P_{13}\right) \Phi_{3}^{(B, 1)}(x), \\
\Pi_{[40]}^{B \neq \Lambda}(x) & =-\frac{1}{2} \Phi_{3}^{(B, 2)}(x),
\end{aligned}
$$

where $P_{13}$ is the permutation operator and

$$
\begin{aligned}
\Phi_{[40]+}^{\Lambda}(x) & =\sqrt{\frac{1}{6}}\left(1+P_{13}\right) \Phi_{3}^{(\Lambda, 1)}(x), \\
\Phi_{[40]+}^{\Lambda}(x) & =-\sqrt{\frac{3}{2}}\left(1-P_{13}\right) \Phi_{3}^{(\Lambda, 1)}(x), \\
\Pi_{[40]}^{\Lambda}(x) & =-\sqrt{\frac{3}{2}} \Phi_{3}^{(\Lambda, 2)}(x) .
\end{aligned}
$$

The normalization factors in Eqs. (A2) and (A3) ensure that in the limit of $S U(3)$ flavor symmetry the following relations hold $[40,45]$ :

$$
\begin{aligned}
& \Phi_{[40]+}^{p}=\Phi_{[40]+}^{\Sigma^{-}}=\Phi_{[40]+}^{\Xi^{0}}=\Phi_{[40]+}^{\Lambda}=\Pi_{[40]}^{\Sigma^{-}}=\Pi_{[40]}^{\Xi^{0}}, \\
& \Phi_{[40]_{-}}^{p}=\Phi_{[40]_{-}}^{\Sigma^{-}}=\Phi_{[40]-}^{\Xi^{0}}=\Phi_{[40]-}^{\Lambda}=\Pi_{[40]}^{\Lambda} .
\end{aligned}
$$




\section{APPENDIX B: POLYNOMIALS}

In this Appendix, we list the lowest polynomials which enter the expansion of the baryon DAs of different twists. We also give the anomalous dimensions of the coefficients that accompany these polynomials.

The twist- 3 polynomials have the forms

$P_{00}(x)=1$,

$P_{10}(x)=\left(x_{1}-x_{3}\right) / 2$,

$P_{11}(x)=\left(x_{1}+x_{3}-2 x_{2}\right) / 2$,

$P_{20}(x)=3 x_{1}^{2}-3 x_{1} x_{2}+2 x_{2}^{2}-6 x_{1} x_{3}-3 x_{2} x_{3}+3 x_{3}^{2}$,

$P_{21}(x)=\left(x_{1}-x_{3}\right)\left(x_{1}+x_{3}-3 x_{2}\right)$,

$P_{22}(x)=x_{1}^{2}+x_{3}^{2}-12 x_{1} x_{3}+9 x_{2}\left(x_{1}+x_{3}\right)-6 x_{2}^{2}$.

The corresponding one-loop anomalous dimensions are (in units of $\left.\alpha_{s} / 2 \pi\right)$

$$
\gamma_{N q}=\left\{\frac{2}{3}, \frac{26}{9}, \frac{10}{3}, \frac{38}{9}, \frac{46}{9}, \frac{16}{3}\right\} .
$$

The mixed chirality twist- 4 polynomials are given by

$$
\begin{aligned}
R_{00}^{-}(x)= & 1, \\
R_{10}^{-}(x)= & x_{1}+x_{3}-\frac{3}{2} x_{2}, \\
R_{10}^{+}(x)= & x_{1}-x_{3}-\frac{1}{2} x_{2}, \\
R_{20}^{+}(x)= & x_{2}^{2}-x_{3}^{2}-2 x_{1} x_{2}+3 x_{1} x_{3}-\frac{2}{3} x_{1}^{2}, \\
\left(\begin{array}{c}
R_{20}^{-}(x) \\
R_{21}^{-}(x)
\end{array}\right)= & x_{2}^{2}+\frac{4}{9}(-5 \pm \sqrt{43}) x_{2} x_{3}+x_{3}^{2} \\
& +\frac{2}{9}(1 \mp 2 \sqrt{43}) x_{1} x_{2}-\frac{1}{9}(17 \pm 2 \sqrt{43}) x_{1} x_{3} \\
& +\frac{4}{27}(4 \pm \sqrt{43}) x_{1}^{2} .
\end{aligned}
$$

The corresponding anomalous dimensions are [38]

$$
\gamma_{N q}=\left\{-2, \frac{2}{9}, 2, \frac{32}{9}, \frac{2(14-\sqrt{43})}{9}, \frac{2(14+\sqrt{43})}{9}\right\} .
$$

The chiral twist- 4 polynomials take the form
$\Pi_{00}(x)=1$,

$\Pi_{10}(x)=x_{1}+x_{3}-\frac{3}{2} x_{2}$,

$\Pi_{20}(x)=x_{1}^{2}-4 x_{1} x_{2}+2 x_{2}^{2}+2 x_{1} x_{3}-4 x_{2} x_{3}+x_{3}^{2}$.

These polynomials correspond to $\gamma_{N q}=\{-2,4 / 3,4 / 3\}$. Finally, for the polynomials $\Lambda^{ \pm}$and $\Pi^{+}$we get

$$
\begin{aligned}
\Lambda_{10}^{(-)}(x) & =x_{2}-x_{3} \\
\Lambda_{20}^{(-)}(x) & =x_{2}^{3}-x_{3}^{2}-6 x_{1}\left(x_{2}-x_{3}\right) \\
\Pi_{20}^{(+)}(x) & =x_{1}^{2}+\frac{1}{3}\left(x_{2}^{3}+x_{3}^{2}\right)-\frac{1}{2} x_{2} x_{3}-x_{1}\left(x_{2}+x_{3}\right)
\end{aligned}
$$

with the anomalous dimensions $\gamma_{N q}=\{-2 / 3,4,4\}$.

We also write down here the first nonzero terms of the WW contributions for the mixed chirality DAs. For the twist-4 DAs one gets

$$
\begin{aligned}
\Phi_{4}^{(B, A) W W}(x)= & \frac{1}{6} \phi_{00}^{(B, A)} x_{1} x_{2}\left(1-2 x_{3}\right)+\ldots, \\
\Phi_{4}^{(B, A) W W}(x)= & \frac{\phi_{10}^{(B, A)}}{24} x_{1} x_{2}\left(x_{1}\left(1-3 x_{3}\right)-x_{3}\left(2-3 x_{3}\right)\right) \\
& +\ldots
\end{aligned}
$$

for the positive and negative signature DAs, respectively. For the twist- 5 functions one gets

$$
\begin{aligned}
\Phi_{5}^{(B, A) W W_{3}}(x)= & \frac{\phi_{00}^{(B, A)}}{6} x_{3}\left(1-2 x_{1}-x_{2}\left(1+2 x_{1}\right)\right)+\ldots, \\
\Phi_{5}^{(B, A) W W_{4}}(x)= & \frac{\eta_{10}^{(B, A)}}{3} x_{3}\left(1-2 x_{3}-x_{2}\left(1+x_{1}-3 x_{3}\right)\right) \\
& +\ldots
\end{aligned}
$$

for the positive signature and

$$
\begin{aligned}
\Phi_{5}^{(B, A) W W_{3}}(x)= & \frac{1}{12} \phi_{10}^{(B, A)} x_{3}\left(x_{1}-\frac{1}{2} x_{3}+x_{2}\left(x_{3}-2 x_{1}\right)\right. \\
& \left.+\frac{3}{2} x_{1}\left(x_{3}-x_{1}\right)+\frac{3}{2} x_{1} x_{2}\left(x_{3}-x_{1}\right)\right)+\ldots, \\
\Phi_{5}^{(B, A) W W_{4}}(x)= & -\frac{1}{3} \eta_{00}^{(B, A)} x_{3}\left(1-x_{2}\right)+\ldots
\end{aligned}
$$

for the negative signature DAs. 
[1] V. L. Chernyak and A. R. Zhitnitsky, Pis'ma Zh. Eksp. Teor. Fiz. 25, 544 (1977) [JETP Lett. 25, 510 (1977)].

[2] V. L. Chernyak, A. R. Zhitnitsky, and V. G. Serbo, Pis'ma Zh. Eksp. Teor. Fiz. 26, 760 (1977) [JETP Lett. 26, 594 (1977)].

[3] V. L. Chernyak, A. A. Ogloblin, and I. R. Zhitnitsky, Yad. Fiz. 48, 1410 (1988) [Z. Phys. C 42, 569 (1989)]; Sov. J. Nucl. Phys. 48, 896 (1988).

[4] A. V. Efremov and A. V. Radyushkin, Phys. Lett. 94B, 245 (1980).

[5] A. V. Efremov and A. V. Radyushkin, Teor. Mat. Fiz. 42, 147 (1980) [Theor. Math. Phys. 42, 97 (1980)].

[6] G. P. Lepage and S. J. Brodsky, Phys. Lett. 87B, 359 (1979).

[7] A. Duncan and A.H. Mueller, Phys. Rev. D 21, 1636 (1980).

[8] A. Duncan and A. H. Mueller, Phys. Lett. 90B, 159 (1980).

[9] A. I. Milshtein and V. S. Fadin, Yad. Fiz. 33, 1391 (1981).

[10] A. I. Milshtein and V. S. Fadin, Yad. Fiz. 35, 1603 (1982).

[11] N. Kivel and M. Vanderhaeghen, Phys. Rev. D 83, 093005 (2011).

[12] J. Bolz, R. Jakob, P. Kroll, M. Bergmann, and N. G. Stefanis, Z. Phys. C 66, 267 (1995).

[13] A. J. R. Puckett et al., Phys. Rev. Lett. 104, 242301 (2010).

[14] I. I. Balitsky, V. M. Braun, and A. V. Kolesnichenko, Yad. Fiz. 44, 1582 (1986) [Sov. J. Nucl. Phys. 44, 1028 (1986)].

[15] I. I. Balitsky, V. M. Braun, and A. V. Kolesnichenko, Nucl. Phys. B312, 509 (1989).

[16] V. L. Chernyak and I. R. Zhitnitsky, Nucl. Phys. B345, 137 (1990).

[17] V. M. Braun, A. Lenz, N. Mahnke, and E. Stein, Phys. Rev. D 65, 074011 (2002).

[18] V. M. Braun, A. Lenz, and M. Wittmann, Phys. Rev. D 73, 094019 (2006).

[19] A. Lenz, M. Wittmann, and E. Stein, Phys. Lett. B 581, 199 (2004).

[20] T. M. Aliev, K. Azizi, A. Ozpineci, and M. Savci, Phys. Rev. D 77, 114014 (2008).

[21] K. Passek-Kumericki and G. Peters, Phys. Rev. D 78, 033009 (2008).
[22] I. V. Anikin, V. M. Braun, and N. Offen, Phys. Rev. D 88, 114021 (2013).

[23] V. Braun, R. J. Fries, N. Mahnke, and E. Stein, Nucl. Phys. B589, 381 (2000); B607, 433(E) (2001).

[24] S. Wandzura and F. Wilczek, Phys. Lett. 72B, 195 (1977).

[25] P. Ball and V. M. Braun, Nucl. Phys. B543, 201 (1999).

[26] P. Ball, V. M. Braun, and A. Lenz, J. High Energy Phys. 05 (2006) 004.

[27] A. V. Belitsky and D. Mueller, Nucl. Phys. B589, 611 (2000).

[28] A. V. Radyushkin and C. Weiss, Phys. Lett. B 493, 332 (2000).

[29] N. Kivel, M. V. Polyakov, A. Schafer, and O. V. Teryaev, Phys. Lett. B 497, 73 (2001).

[30] I. V. Anikin and O. V. Teryaev, Phys. Lett. B 509, 95 (2001).

[31] B. Geyer, D. Robaschik, and J. Eilers, Nucl. Phys. B704, 279 (2005).

[32] O. V. Teryaev, arXiv:hep-ph/0102296.

[33] J. Blumlein and N. Kochelev, Nucl. Phys. B498, 285 (1997).

[34] J. Blumlein and A. Tkabladze, Nucl. Phys. B553, 427 (1999).

[35] H. Avakian, A. V. Efremov, K. Goeke, A. Metz, P. Schweitzer, and T. Teckentrup, Phys. Rev. D 77, 014023 (2008).

[36] A. Metz, P. Schweitzer, and T. Teckentrup, Phys. Lett. B 680, 141 (2009).

[37] A. Accardi, A. Bacchetta, W. Melnitchouk, and M. Schlegel, J. High Energy Phys. 11 (2009) 093.

[38] V. M. Braun, A. N. Manashov, and J. Rohrwild, Nucl. Phys. B807, 89 (2009).

[39] I. V. Anikin and A. N. Manashov, Phys. Rev. D 89, 014011 (2014).

[40] G. S. Bali et al., arXiv:1512.02050.

[41] V. M. Braun, T. Lautenschlager, A. N. Manashov, and B. Pirnay, Phys. Rev. D 83, 094023 (2011).

[42] M. Bergmann, W. Schroers, and N. G. Stefanis, Phys. Lett. B 458, 109 (1999).

[43] N. G. Stefanis, Eur. Phys. J. direct C 7, 1 (1999).

[44] V. M. Braun, S. E. Derkachov, G. P. Korchemsky, and A. N. Manashov, Nucl. Phys. B553, 355 (1999).

[45] P. Wein and A. Schäfer, J. High Energy Phys. 05 (2015) 073. 